\title{
Genetic risk scores, sex and dietary factors interact to alter serum uric acid trajectory among African-American urban adults
}

\author{
May A. Beydoun ${ }^{1 *}$, Jose-Atilio Canas $^{2}$, Marie T. Fanelli-Kuczmarski ${ }^{3}$, Salman M. Tajuddin ${ }^{1}$, \\ Michele K. Evans ${ }^{1} \dagger$ and Alan B. Zonderman ${ }^{1} \dagger$ \\ ${ }^{1}$ Laboratory of Epidemiology and Population Sciences, National Institute on Aging (NIA), National Institutes of Health, \\ Intramural Research Program (NIH/IRP), Baltimore, MD 21224, USA \\ ${ }^{2}$ Pediatric Endocrinology, Diabetes and Metabolism, Nemour's Children's Clinic, Jacksonville, FL 32207, USA \\ ${ }^{3}$ Department of Behavioral Health and Nutrition, University of Delaware, Newark, DE 19716, USA
}

(Submitted 26 July 2016 - Final revision received 5 December 2016 - Accepted 3 February 2017 - First published online 27 March 2017)

\section{Abstract}

Serum uric acid (SUA), a causative agent for gout among others, is affected by both genetic and dietary factors, perhaps differentially by sex. We evaluated cross-sectional (SUA base) and longitudinal (SUA rate) associations of SUA with a genetic risk score (GRS), diet and sex. We then tested the interactive effect of GRS, diet and sex on SUA. Longitudinal data on 766 African-American urban adults participating in the Healthy Aging in Neighborhood of Diversity across the Lifespan study were used. In all, three GRS for SUA were created from known SUA-associated SNP (GRS base $(n 12 \mathrm{SNP}), \mathrm{GRS}_{\text {rate }}(n 3 \mathrm{SNP})$ and $\left.\mathrm{GRS}_{\text {total }}(n 15 \mathrm{SNP})\right)$. Dietary factors included added sugar, total alcohol, red meat, total fish, legumes, dairy products, caffeine and vitamin C. Mixed-effects linear regression models were conducted. SUA base $_{\text {was }}$ higher among men compared with that among women, and increased with $\mathrm{GRS}_{\text {total }}$ tertiles. SUA rate was positively associated with legume intake in women $(\gamma=+0 \cdot 14 ; 95 \% \mathrm{CI}+0 \cdot 06,+0 \cdot 22, P=0 \cdot 001)$ and inversely related to dairy product intake in both sexes combined $(\gamma=-0 \cdot 042 ; 95 \% \mathrm{CI}-0 \cdot 075$, $-0 \cdot 009), P=0 \cdot 010)$. SUA base $_{\text {bas }}$ directly linked to alcohol consumption among women $(\gamma=+0 \cdot 154 ; 95 \% \mathrm{CI}+0 \cdot 046,+0 \cdot 262, P=0 \cdot 005)$. GRS rate was linearly related to $\mathrm{SUA}_{\text {rate }}$ only among men. Legume consumption was also positively associated with SUA rate within the GRS $_{\text {total }}$ 's lowest tertile. Among women, a synergistic interaction was observed between GRS ${ }_{\text {rate }}$ and red meat intake in association with SUA rate Among men, a synergistic interaction between low vitamin $\mathrm{C}$ and genetic risk was found. In sum, sex-diet, sex-gene and gene-diet interactions were detected in determining SUA. Further similar studies are needed to replicate our findings.

\section{Key words: Serum uric acid: Diets: Genetic risk scores: African-Americans: Urban adults}

Uric acid (UA), the final catabolic product of purine oxidation, is the causative agent of gout, characterised by urate crystal deposition in joints and elevated serum uric acid (SUA) or hyperuricaemia $^{(1)}$. Gout affects $6-8 \%$ of the elderly ( $>80$ years) and approximately $3.9 \%$ of the entire US population ${ }^{(2)}$. Moreover, hyperuricaemia independently predicts myocardial infarction and premature death ${ }^{(3)}$. Two key physiological mechanisms determining hyperuricaemia are increased liver production of urate from dietary and endogenous substrates that raise purine levels, and reduced renal and gut excretion of $\mathrm{UA}^{(4)}$. Thus, uncovering a genetic basis for both mechanisms might elucidate the aetiological factors behind gout. Recent genome-wide association studies (GWAS) have identified various genetic loci with the strongest influences on SUA such as ATP binding cassette subfamily $\mathrm{G}$ member 2 (ABCG2), sodium/phosphate cotransporter 4 (NPT4) (solute carrier family 17 (organic anion transporter), member 3), NPT1 (solute carrier family 17 (organic anion transporter), member 1 (SLC17A1)), solute carrier family 22 (organic anion/urate transporter), member 12 (URAT1) (solute carrier family 22 (organic anion/ urate transporter), member 12 (SLC22A12)), organic anion uptake transporter 4 (OAT4) (solute carrier family 22 (organic anion/urate transporter), member 11) and GLUT9 (solute carrier family 2 (facilitated GLUT), member $9(S L C 2 A 9))^{(2)}$ However, no study thus far has compiled all recently identified SNP into a genetic risk score (GRS) for SUA in a longitudinal study of African-American (AA) adults. Moreover, the sex-specific effect of this GRS is yet to be uncovered.

Although genetics has a strong influence on SUA, dietary factors including the Mediterranean Diet Score ${ }^{(5,6)}$ and specific components may have equally important effects ${ }^{(2)}$. On the basis of recent data ${ }^{(1,7-12)}$, it is hypothesised that red meat and seafood consumption are linked to an increased risk for gout and/or hyperuricaemia ${ }^{(1,9)}$, with similar adverse effects found

Abbreviations: AA, African-American; GRS, genetic risk score; HANDLS, Healthy Aging in Neighborhoods of Diversity Across the Lifespan; SLC2A9, solute carrier family 2 (facilitated GLUT), member 9; SUA, serum uric acid; UA, uric acid.

* Corresponding author: M. A. Beydoun, fax +1 410558 8236, email baydounm@mail.nih.gov

$\dagger$ Co-senior authors. 
for alcohol, particularly from beer and liquor ${ }^{(1,7,9,10,13,14)}$, and fructose-containing foods including soft drinks ${ }^{(1,9,11,12)}$ as well as intake of legumes in animal studies ${ }^{(15)}$. In contrast, dairy products, particularly low-fat milk and yogurt ${ }^{(1,9,10,14)}$ intake, caffeine intake $e^{(1,9,14)}$ and vitamin $C^{(1,9,14)}$ intake are all hypothesised to be inversely related to gout and/or hyperuricaemia risk. This study evaluated the cross-sectional ( $\left.\mathrm{SUA}_{\text {base }}\right)$ and longitudinal ( $\mathrm{SUA}_{\text {rate }}$ ) associations of SUA with GRS, diet and sex. We then tested interactive effect of GRS, diet and sex on SUA.

Thus, using genetic data available on the AA urban adults participating in the Healthy Aging in Neighborhoods of Diversity Across the LifeSpan (HANDLS), this study had several key aims: first, the study generates and evaluates the effects of GRS for elevated SUA by relating it to both baseline SUA and over-time change in SUA among AA urban adults. Second, the study examines sex-specific association between this GRS and SUA, an association previously observed in individual $\mathrm{SNP}^{(16-18)}$. Finally, the study evaluates the relationship between the eight previously described dietary factors and SUA at baseline and change over time, while examining sex-diet interactions and gene-diet interactions within sex groups.

\section{Methods}

\section{Database}

HANDLS is a prospective cohort study of a representative sample of AA and White men and women aged 30-64 years at baseline. Details of the study design have been described previously $^{(23)}$ (http://handls.nih.gov/). In brief, data were collected in two separate phases at baseline (2004-2009; visit 1), with Phase 1 assessing socio-demographic information (age, sex, education, poverty status, etc.), physiological and psychological chronic exposure, and including the first 24-h dietary recall, whereas Phase 2 consisting of in-depth examinations in Mobile Research Vehicles and including a second 24-h dietary recall, psychometric, anthropometric, body composition and laboratory parameter measurements ${ }^{(19)}$. Visit 2 of HANDLS, initiated in 2009, followed a similar protocol, from which laboratory measurements, specifically SUA, were utilised in this study.

Procedures followed the ethical standards of the institution and approval was obtained from The MedStar Institutional Review Board, and written informed consent was obtained from all HANDLS participants.

\section{Study sample}

Data were derived from baseline visit 1 (2004-2009) and the first follow-up examination (visit 2; 2009-2013), and were appended in the long format to facilitate mixed-effects regression modelling analyses ( $N$ is the number of persons, $N^{\prime}$ the number of observations and $k$ the number of observations/person). Follow-up time (range: <1-approximately 8 years) had a mean of 4.64 (SD 0.93) years, with time $=0$ for the baseline visit and time $=$ elapsed years to the nearest day for follow-up visit. HANDLS initially recruited $N_{1} 3720$ participants (sample 1, $n_{1} 2198 \mathrm{AA}$ ), with total observations at both visits being $N_{1}{ }^{\prime} 6025\left(n_{1}{ }^{\prime} 3616 \mathrm{AA}\right)$.
Among all HANDLS participants, SUA was available at either visits 1 or 2 for $N_{2} 3021$ ( $\left.N_{2}{ }^{\prime} 5315\right)$, of whom $n_{2} 1,792$ were AA with $n_{2}^{\prime} 3199$ observations (sample 2). Of AA in sample 2, participants with missing data on any of the two baseline 24-h dietary recalls were excluded, yielding a sample size of $n_{3} 1235\left(n_{3}{ }^{\prime} 2206\right)$ (sample 3). Out of these participants, only those with complete genetic data (original sample, $n 1024$ AA) were selected ( $n_{4} 766$; $n_{4}^{\prime} 1375$; visits/person, $k$ 1.8) (sample 4). Thus, our final sample consisted of AA with complete genetic data, complete baseline dietary data with two 24-h recalls and SUA measured at either of the two visits. Sample 4 differed from the unselected participants of sample $1 \mathrm{AA}$, by having a lower proportion above poverty ( $49.5 v .54 .3 \%, P=0.032$ ), with no notable differences by sex or age (online Supplementary Fig. S1). The same pattern was noted when comparing AA with complete genetic data who were selected ( $n_{4} 766,49.5 \%$ above poverty) to those who were not (n 258/1024, 62.8\% above poverty).

\section{Serum uric acid}

Using $1 \mathrm{ml}$ of fasting blood serum, SUA was measured using a standard spectrophotometry method at both visits (Quest Diagnostics） (http://www.questdiagnostics.com/testcenter/ TestDetail.action?ntc=905). SUA was measured at both visits in HANDLS, and expressed in $\mathrm{mg} / \mathrm{dl}$, whereby $1 \mathrm{mg} / \mathrm{dl}$ of SUA is equivalent to $0 \cdot 01681237 \mu \mathrm{mol} / 1$.

\section{Dietary assessment}

Dietary factors included in our analyses were measured at the baseline visit. Both baseline 24-h dietary recalls were obtained using the US Department of Agriculture Automated Multiple Pass Method, a computerised structured interview ${ }^{(20)}$. Measurement aids were used and included measuring cups, spoons, a ruler and an illustrated Food Model Booklet. Both recalls were administered in-person by trained interviewers, 4-10 d apart. Trained nutrition professionals used Survey Net, matching foods consumed with eight-digit codes from the Food and Nutrient Database for Dietary Studies version 3.0 ${ }^{(21)}$, and MyPyramid Equivalents Database (MPED) for food groups (MPED 2: http://www.ars.usda.gov/SP2UserFiles/Place/80400530/ $\mathrm{pdf} / \mathrm{mped} / \mathrm{mped} 2$ doc.pdf). Eight dietary factors were chosen as proxy or direct measures for dietary components previously linked SUA: (1) added sugars (teaspoon/d), (2) alcoholic beverages (drinks/d, with one drink defined as twelve fluid ounces of beer, five fluid ounces of wine, or one-and-a-half fluid ounces of 80-proof distilled spirits), (3) ounce equivalents/d of red meats, (4) ounce equivalents/d of fish (sum of fish high and low in $n-3$ fatty acids), and (5) cup equivalents/d of legumes, (6) cup equivalents/d of dairy products (milk, cheese and yogurt), (7) dietary vitamin $\mathrm{C}$ from foods $(\mathrm{mg} / \mathrm{d})$, and (8) caffeine $(\mathrm{g} / \mathrm{d})$; the later three were associated with reduced SUA ${ }^{(1,9)}$.

\section{Serum uric acid-genetic risk score construction}

Genotyping was performed in 1024 HANDLS AA participants using Illumina 1M SNP genotyping array (online Supplementary Appendix S1). A high-quality review paper of GWAS studies examining SNP at various gene loci in relation to phenotypes 
of SUA, gout or hyperuricaemia was used as a starting point for listing the SNP in the online Supplementary Table $S 1^{(22)}$. This list was updated with four more recent GWAS studies ${ }^{(22-26)}$. Despite the paucity of studies in AA adults, all SNP were included in the pool of potentially influential polymorphisms prospectively affecting SUA in our AA urban sample. Genotypes were imputed using the 1000 Genomes Project phase 1 multiethnic reference panel, with SNP extracted only from high-quality imputed genotypes. Of sixty-eight SNP, four were unavailable and rs72552713 was excluded because of poor imputation quality (imputation quality $R^{2}$ 0.0073). After performing linkage disequilibrium (LD)-based SNP pruning, using an LD threshold $R^{2}$ of 0.8 in a $500 \mathrm{~kb}$ sliding window, forty-three independent markers were selected for further analysis. Using mixed-effects regression models adjusted for sociodemographic and lifestyle variables, dietary factors, ten principal components (PC) and the inverse Mills ratio, the forty-three SNP were screened for significant effects on SUA at baseline and rate of change in SUA at a type I error rate of $0 \cdot 10$ (online Supplementary Appendix S2 and Table S2). Only fifteen of the forty-three showed a significant association with baseline SUA ( $n$ 12) or rate of change in SUA ( $n$ 3). Those fifteen SNP were used to construct three GRS, one for total ( $\left.\mathrm{GRS}_{\text {total }}, n 15\right)$, one for baseline $\left(\mathrm{GRS}_{\text {base }}, n 12\right)$, and one for rate of change $\left(\mathrm{GRS}_{\text {rate }}\right.$, $n$ 3). Given the marked difference in interpretation of effects (base $v$. rate), only unweighted GRS were constructed and could range from 0 to 30 for $\mathrm{GRS}_{\text {total }}, 0$ to 24 for $\mathrm{GRS}_{\text {base }}$ and 0 to 6 for $\mathrm{GRS}_{\text {rate. }}$. The online Supplementary Table S1 describes those SNP, along with the selection process leading to the three GRS. The online Supplementary Table S2 shows the results of the mixed-effects regression models of the fifteen selected SNP. Notably, seven of the fifteen selected SNP were located on or near the $S L C 2 A 9$ gene. The remaining eight SNP were located on $A B C G 2$ ( $n$ 1), SLC22A12 ( $n$ 1), SLC17A1 ( $n$ 1), glucokinase (hexokinase 4$)$ regulator $(n 1)$, leucine rich repeat containing $16 \mathrm{~A}(n 1)$, neurexin $2(n 1)$, nuclear factor of activated T-cells 5 , tonicity-responsive $(n 1)$ and hepatic leukaemia factor $(n 1)$.

\section{Covariates}

Covariates included sex, age, education ( $<$ high school (HS) (grades 1-8), HS (grades 9-12), >HS (grade 13+)), poverty status (household incomes below or above $125 \%$ of the 2004 Federal poverty guidelines), smoking status (current smoker $v$. no use of cigarettes), illicit drug use (current $v$. no use of either marijuana, cocaine or opiates), BMI measured as weight/squared measured height $\left(\mathrm{kg} / \mathrm{m}^{2}\right)$ ten $\mathrm{PC}$ to control for population stratification (online Supplementary Table S1) and selected food groups determined using the MPED2 (http://www.ars.usda.gov/SP2 UserFiles/Place/80400530/pdf/mped/mped2_doc.pdf), namely total fruits, total vegetables (cup equivalents/d), total grains (ounce equivalents/d), other meats (ounce equivalents/d) and discretionary solid fats and oils $(\mathrm{g} / \mathrm{d})$.

\section{Statistical methods}

Using Stata 13.0., sampling weights were included only in descriptive analyses, whereby means and proportions were compared across sex and GRS tertiles, using design-based
$F$ test. Moreover, $P_{\text {for trend }}$ values were estimated by entering GRS as an ordinal predictor in a bivariate regression model. Baseline and follow-up SUA were also plotted (box plots) and compared across GRS tertiles and $\operatorname{sex}^{(27)}$. In the main part of the analysis, four sets of time-interval mixed-effects regression models with the outcome SUA measured at either visits 1 or 2 were conducted, which assumes missingness at random ${ }^{(28)}$. (online Supplementary Appendix S2)

In a first model set, eight dietary components predicted baseline SUA ( $\mathrm{SUA}_{\text {base }}$ ) and annual rate of change in SUA $\left(\mathrm{SUA}_{\text {rate }}\right)$, overall and stratifying by sex. Type I error in analyses examining dietary factors was corrected for multiple testing using Bonferroni correction, assuming an initial type I error rate of 0.05 for main effects and 0.10 for interaction terms, yielding a corrected error rates of $0.05 / 8=0.006$ and $0 \cdot 10 / 8=0.013$, respectively ${ }^{(29,30)}$.

In a second model set, the GRS uppermost two tertiles were contrasted with the lowest in their association with SUA base (cross-sectional, exposure main effect, $\mathrm{GRS}_{\text {base }}$ ) and $\mathrm{SUA}_{\text {rate }}$ (longitudinal, exposure $\times$ Time, GRS $_{\text {rate }}$ ); (model A). Crosssectional and longitudinal effects were compared between sexes and tested for effect modification by including two-way and three-way interactions with sex in unstratified models. In

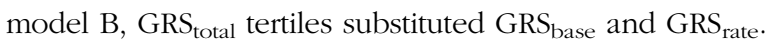

In a third model set, eight dietary factors were also of primary interest, while effect modification was tested for $\mathrm{GRS}_{\text {total }}$ tertiles, by adding two-way and three-way interaction terms in the unstratified model.

Finally, stratifying the analysis by sex, gene-diet interactions were tested in a fourth model set, whereby each of eight dietary factors were separately interacted with continuous $\mathrm{GRS}_{\text {base }}$ to test their interactive effects on SUA base. Similarly, three-way interactions between each dietary component, Time and continuous $\mathrm{GRS}_{\text {rate }}$ were also examined in separate models. Predictive margins were estimated and plotted across Time, stratifying by exposure group, from selected mixed-effects regression models.

Selection bias due to the non-random selection of participants with complete data was corrected for, using a two-stage Heckman selection process, as was done in other previous studies ${ }^{(31,32)}$.

\section{Results}

Table 1 describes baseline characteristics of the study sample by sex and by GRS tertile. While $55.2 \%$ of the sample consisted of women, mean age overall was estimated at 47.4 years. Being below poverty was more likely in women, whereas being a current illicit drug user was more likely in men. Women also had a higher mean BMI than men. Men consumed higher amounts of all selected dietary factors than women, except for fish, caffeine and total vegetables (Table 1). Men had higher SUA at both baseline and follow-up compared with women, and there was a consistent positive association between $\mathrm{GRS}_{\text {total }}$ tertiles and SUA (baseline and follow-up). (online Supplementary Fig. S2 and S3)

Several key findings emerged from the mixed-effects regression models (Tables 2-5). After correction for multiple testing, overall, (Table 2; online Supplementary Fig. S4), higher 
Table 1. Baseline study characteristics by sex and genetic risk score (GRS) tertile (T), Healthy Aging in Neighborhoods of Diversity Across the Lifespan (Mean values with their standard errors)

\begin{tabular}{|c|c|c|c|c|c|c|c|c|c|c|c|c|c|c|}
\hline & & & \multicolumn{4}{|c|}{ Sex } & \multirow[b]{3}{*}{$P^{*}$} & \multicolumn{6}{|c|}{$\mathrm{GRS}_{\text {total }}$ tertile } & \multirow[b]{3}{*}{$P_{\text {for trend }} \dagger$} \\
\hline & \multicolumn{2}{|c|}{ Total $(n 766)$} & \multicolumn{2}{|c|}{ Men $(n 343)$} & \multicolumn{2}{|c|}{ Women $(n$ 423) } & & \multicolumn{2}{|c|}{$\mathrm{T} 1$} & \multicolumn{2}{|c|}{$\mathrm{T} 2$} & \multicolumn{2}{|c|}{ T3 } & \\
\hline & Mean & SEM & Mean & SEM & Mean & SEM & & Mean & SEM & Mean & SEM & Mean & SEM & \\
\hline Age (years) & $47 \cdot 4$ & 0.6 & $48 \cdot 4$ & 0.8 & $46 \cdot 6$ & 0.8 & 0.14 & $45 \cdot 7$ & 1.1 & $48 \cdot 2$ & 0.7 & $48 \cdot 8$ & $1 \cdot 2$ & 0.043 \\
\hline Marital status (\%) & \multirow{2}{*}{\multicolumn{2}{|c|}{27.5}} & & & & & 0.23 & & & & & & & 0.81 \\
\hline Married & & & \multicolumn{2}{|c|}{$30 \cdot 2$} & \multicolumn{2}{|c|}{$25 \cdot 1$} & & \multicolumn{2}{|c|}{23.9} & \multicolumn{2}{|c|}{29.7} & \multicolumn{2}{|c|}{$29 \cdot 1$} & \\
\hline Missing & \multicolumn{2}{|c|}{3.6} & 4 & & \multicolumn{2}{|c|}{2.5} & & \multicolumn{2}{|c|}{3.7} & \multicolumn{2}{|c|}{3.7} & \multicolumn{2}{|c|}{3.0} & \\
\hline Education (\%) & & & & & & & 0.53 & & & & & & & 0.25 \\
\hline$<$ High school & \multicolumn{2}{|c|}{3.4} & 2 & & \multicolumn{2}{|c|}{4.0} & & \multicolumn{2}{|c|}{3.0} & 3 & 0 & & .7 & \\
\hline High school & 59 & & 57 & & & & & 69 & & 54 & & 55 & & \\
\hline > High school & 37 & & 40 & & & & & 28 & & 43 & & 40 & & \\
\hline Poverty:income ratio $<125 \%$, (\%) & 23 & & 18 & & & & 0.015 & 24 & & 23 & & 21 & & 0.83 \\
\hline Current smoking status (\%) & & & & & & & 0.08 & & & & & & & 0.07 \\
\hline Yes & 48 & & 56 & & & & & 55 & & 39 & & 52 & & \\
\hline Missing & 6 & & 4 & & & & & 4 & & 10 & & & .9 & \\
\hline Current illicit drug use (\%) & & & & & & & 0.009 & & & & & & & 0.09 \\
\hline Yes & 23 & & 31 & & & & & 29 & & 18 & & 23 & & \\
\hline Missing & 4 & & 2 & & & & & 1 & & 8 & 8 & & $\cdot 1$ & \\
\hline $\operatorname{BMI}\left(\mathrm{kg} / \mathrm{m}^{2}\right)$ & 29.4 & 0.5 & $27 \cdot 3$ & 0.5 & $31 \cdot 2$ & 0.8 & $<0.001$ & 28.5 & 0.8 & $30 \cdot 0$ & 0.8 & 29.6 & $1 \cdot 1$ & 0.36 \\
\hline Key dietary intake factors & & & & & & & & & & & & & & \\
\hline Added sugars (teaspoon/d) & $22 \cdot 6$ & $1 \cdot 2$ & $25 \cdot 3$ & 1.7 & $20 \cdot 2$ & 1.6 & 0.031 & $25 \cdot 9$ & $2 \cdot 2$ & $21 \cdot 2$ & 1.8 & $20 \cdot 0$ & 1.6 & 0.029 \\
\hline Alcoholic beverages (drinks/d) & 0.70 & 0.1 & 1.13 & 0.2 & 0.32 & 0.06 & $<0.001$ & 0.4 & 0.1 & 0.9 & 0.2 & 0.8 & 0.2 & 0.08 \\
\hline Red meat (oz equiv/d) & 1.70 & 0.15 & $2 \cdot 25$ & 0.25 & 1.22 & 0.17 & 0.001 & $2 \cdot 1$ & 0.3 & 1.5 & 0.2 & 1.5 & 0.2 & 0.09 \\
\hline Fish (oz equiv/d) & 1.12 & 0.15 & $1 \cdot 13$ & 0.17 & $1 \cdot 10$ & 0.23 & 0.92 & 1.2 & 0.2 & 1.2 & 0.3 & 0.9 & 0.2 & 0.42 \\
\hline Legumes (cup equiv/d) & 0.04 & 0.01 & 0.06 & 0.02 & 0.02 & 0.06 & 0.034 & 0.02 & 0.01 & 0.06 & 0.02 & 0.04 & 0.01 & 0.13 \\
\hline Dairy products (cups equiv/d) & 0.96 & 0.07 & $1 \cdot 15$ & 0.10 & 0.79 & 0.08 & 0.008 & 1.03 & 0.13 & 0.95 & 0.09 & 0.85 & 0.13 & 0.33 \\
\hline Vitamin C $(\mathrm{mg} / \mathrm{d})$ & 83.7 & $5 \cdot 2$ & 99.9 & 8.6 & 69.4 & $5 \cdot 2$ & 0.003 & 79.1 & $7 \cdot 6$ & 89.0 & 8.6 & 81.9 & 11.5 & 0.75 \\
\hline Caffeine $(\mathrm{mg} / \mathrm{d})$ & $76 \cdot 4$ & $5 \cdot 0$ & $80 \cdot 8$ & $7 \cdot 3$ & 72.5 & 6.9 & 0.40 & 84.2 & 9.5 & 63.0 & $7 \cdot 2$ & $87 \cdot 3$ & $8 \cdot 2$ & 0.96 \\
\hline Other dietary intake factors & & & & & & & & & & & & & & \\
\hline Total grains (oz equiv/d) & 5.90 & 0.21 & $6 \cdot 82$ & 0.33 & 5.09 & 0.23 & $<0.001$ & $6 \cdot 0$ & 0.3 & $5 \cdot 7$ & 0.3 & $6 \cdot 2$ & 0.5 & 0.79 \\
\hline Total fruits (cup equiv/d) & 0.77 & 0.05 & 0.93 & 0.08 & 0.62 & 0.06 & 0.003 & 0.7 & 0.1 & 0.8 & 0.1 & 0.9 & 0.1 & 0.40 \\
\hline Total vegetables (cup equiv/d) & 1.39 & $0 \cdot 10$ & 1.54 & 0.19 & 1.25 & 0.07 & 0.15 & 1.3 & 0.1 & 1.5 & 0.2 & 1.3 & 0.1 & 0.62 \\
\hline Other meats (oz equiv/d) & 4.76 & 0.22 & $5 \cdot 92$ & 0.37 & 3.73 & 0.21 & $<0.001$ & $5 \cdot 0$ & 0.4 & $4 \cdot 3$ & 0.3 & $5 \cdot 0$ & 0.4 & 0.83 \\
\hline Discretionary oil $(\mathrm{g} / \mathrm{d})$ & 18.04 & 1.56 & 22.04 & 3.03 & 14.49 & 0.95 & 0.017 & 14.9 & 1.5 & $20 \cdot 1$ & $2 \cdot 5$ & $19 \cdot 2$ & 4.3 & 0.26 \\
\hline Discretionary solid fat $(\mathrm{g} / \mathrm{d})$ & 47.45 & 1.96 & $58 \cdot 26$ & $2 \cdot 84$ & 37.87 & $2 \cdot 37$ & $<0.001$ & 51.7 & 3.6 & $45 \cdot 6$ & 2.9 & $44 \cdot 3$ & 3.5 & 0.13 \\
\hline
\end{tabular}

* $P$ value for null hypothesis of no sex difference based on a design-based $F$ test.

$\dagger P_{\text {for trend }}$ value was based on design-based $F$ test for trend in exposures across tertiles of GRS.

rate of change in SUA was associated with lower dairy product intake $\left(\gamma_{16}-0.042 ; 95 \% \mathrm{CI}-0.075,-0.009, P=0 \cdot 010\right)$ When examining sex-specific associations, the association of legume intake with $\mathrm{SUA}_{\text {rate }}$ was stronger among women $(\gamma+0 \cdot 14 ; 95 \%$ $\mathrm{CI}+0 \cdot 06,+0 \cdot 22, P=0 \cdot 001)$, while alcohol intake was positively associated with SUA $_{\text {base }}$ also among women $(\gamma+0 \cdot 154 ; 95 \% \mathrm{CI}$ $+0 \cdot 046,+0 \cdot 262, P=0 \cdot 005)$.

Table 3 tests associations between GRS $_{\text {base }}$ tertiles and baseline SUA and between GRS $_{\text {rate }}$ tertiles and rate of change in SUA, overall and stratified by sex (model A). Both the middle and uppermost tertiles of GRS base were associated with higher SUA compared with the lowest tertile, with a significantly stronger association of the highest tertile $v$. lowest among women and the middle tertile $v$. lowest among men. Only the uppermost tertile of $\mathrm{GRS}_{\text {rate }}$ was linked to faster rate of increase in $\mathrm{SUA}_{\text {rate }}$ compared with the lowest tertile. This effect was significantly stronger among men and non-significant in women. The predictive margins of SUA across time by tertiles of $\mathrm{GRS}_{\text {base }}$ and $\mathrm{GRS}_{\text {rate }}$ are presented in Fig. 1(a) and (b). Mixed-effects regression models with $\mathrm{GRS}_{\text {total }}$ tertiles (model B) indicated that higher $\mathrm{GRS}_{\text {total }}$ was associated with higher $\mathrm{SUA}_{\text {base }}$ overall though no association was detected with $\mathrm{SUA}_{\text {rate. }}$
In Table 4, after correction for multiple testing, the association between legume consumption and $\mathrm{SUA}_{\text {rate }}$ was restricted to the lowest tertile of $\mathrm{GRS}_{\text {total; }}\left(\gamma_{15}+0 \cdot 491 ; 95 \% \mathrm{CI}+0 \cdot 246,+0 \cdot 736\right.$, $P<0.001)$, indicating an antagonistic $\mathrm{GRS}_{\text {total }} \times$ legume interaction.

In Table 5, among women, we detected a synergistic interaction between $\mathrm{GRS}_{\text {rate }}$ and red meat consumption in relation to SUA $_{\text {rate }}\left(\gamma_{139}+0.025\right.$ (standard error of the estimate (SEE) 0.010), $P=0 \cdot 012)$. Specifically, $\mathrm{GRS}_{\text {rate }}$ among women was associated with non-significant increase in SUA over time among non-consumers of red meat, which was accelerated with red meat consumption. Among men, lower vitamin $\mathrm{C}$ intake was associated with higher SUA base, particularly at higher $\mathrm{GRS}_{\text {base }}$ $\left(\gamma_{079}+0 \cdot 001\right.$ (SEE 0.000), $\left.P=0 \cdot 006\right)$ indicating also a synergistic effect between having high genetic and high dietary risk in terms of lower vitamin C intake.

\section{Discussion}

To our knowledge, this is the first study to evaluate $\mathrm{SUA}_{\text {base }}$ and $\mathrm{SUA}_{\text {rate }}$ associations with GRS in a large sample of AA urban adults, while examining sex-specific genetic and dietary 
Table 2. Mixed-effects regression models of serum uric acid (SUA) by dietary components, stratified by sex* (Regression coefficients $(\gamma)$ with their standard errors of the estimate (SEE))

\begin{tabular}{|c|c|c|c|c|c|c|c|c|c|}
\hline \multirow[b]{3}{*}{ SUA } & \multicolumn{3}{|c|}{ Total: model $1 \dagger$} & \multicolumn{3}{|c|}{ Men: model $2 \dagger$} & \multicolumn{3}{|c|}{ Women: model 3† } \\
\hline & \multicolumn{2}{|c|}{$n 766$} & \multirow{2}{*}{$\frac{n^{\prime} 1341}{P}$} & \multicolumn{2}{|c|}{$n 343$} & \multirow{2}{*}{$\frac{n^{\prime} 583}{P}$} & \multicolumn{2}{|c|}{$n 423$} & \multirow{2}{*}{$\frac{n^{\prime} 758}{P}$} \\
\hline & $\gamma$ & SEE & & $\gamma$ & SEE & & $\gamma$ & SEE & \\
\hline \multicolumn{10}{|l|}{ Fixed effects } \\
\hline Added sugar $\left(\gamma_{01}\right.$ for $\left.\pi_{0 i}\right)$ & +0.002 & 0.004 & 0.69 & +0.003 & 0.005 & 0.55 & -0.003 & 0.006 & 0.65 \\
\hline Added sugar $\times$ Time $\left(\nu_{11}\right.$ for $\left.\pi_{1 i}\right)$ & +0.0005 & 0.0009 & 0.63 & -0.0002 & 0.001 & 0.90 & $+0.002 \ddagger$ & 0.001 & $0.083 \ddagger$ \\
\hline Alcohol $\left(\gamma_{02}\right.$ for $\left.\pi_{0 i}\right)$ & $+0.082 \S$ & 0.032 & $0.010 \S$ & +0.05 & 0.04 & 0.22 & $+0 \cdot 154 \S \|$ & 0.055 & $0.005 \S \|$ \\
\hline Alcohol $\times$ Time $\left(\gamma_{12}\right.$ for $\left.\pi_{1 i}\right)$ & -0.008 & 0.008 & 0.30 & -0.002 & 0.011 & 0.83 & -0.018 & 0.012 & 0.12 \\
\hline Red meat $\left(\gamma_{03}\right.$ for $\left.\pi_{0 j}\right)$ & $+0.046 \S$ & 0.021 & $0.031 \S$ & +0.035 & 0.025 & 0.15 & $+0.090 \S$ & 0.045 & $0.044 \S$ \\
\hline Red meat $\times$ Time $\left(\gamma_{13}\right.$ for $\left.\pi_{1 i}\right)$ & -0.002 & 0.005 & 0.70 & -0.003 & 0.006 & 0.55 & -0.001 & 0.010 & 0.94 \\
\hline Fish $\left(\gamma_{04}\right.$ for $\left.\pi_{0 i}\right)$ & +0.025 & 0.025 & 0.31 & +0.011 & 0.040 & 0.78 & +0.028 & 0.032 & 0.39 \\
\hline Fish $\times$ Time $\left(\gamma_{14}\right.$ for $\left.\pi_{1 i}\right)$ & -0.004 & 0.006 & 0.44 & -0.002 & 0.010 & 0.87 & -0.006 & 0.007 & 0.39 \\
\hline Legumes $\left(\gamma_{05}\right.$ for $\left.\pi_{0 i}\right)$ & -0.28 & 0.18 & 0.12 & -0.33 & 0.37 & 0.38 & -0.29 & 0.21 & 0.17 \\
\hline Legumes $\times$ Time $\left(\gamma_{15}\right.$ for $\left.\pi_{1 i}\right)$ & $+0.09 \S$ & 0.04 & $0.018 \S$ & -0.073 & 0.087 & $0.40 \pi$ & $+0.14 \S \|$ & 0.04 & $0.001 \S \|$ \\
\hline Dairy products $\left(\gamma_{06}\right.$ for $\left.\pi_{0 i}\right)$ & +0.09 & 0.07 & 0.24 & +0.07 & 0.11 & 0.50 & +0.15 & 0.11 & 0.17 \\
\hline Dairy products $\times$ Time $\left(\gamma_{16}\right.$ for $\left.\pi_{1 i}\right)$ & $-0.042 \S \|$ & 0.017 & $0.010 \S ॥$ & -0.037 & 0.025 & 0.14 & $-0.057 \S$ & 0.024 & $0.015 \S$ \\
\hline Vitamin C $\left(\gamma_{07}\right.$ for $\left.\pi_{0 i}\right)$ & -0.001 & 0.001 & 0.12 & $-0.003 \S$ & 0.001 & $0.044 \S$ & -0.001 & 0.001 & 0.71 \\
\hline Vitamin C $\times$ Time $\left(\gamma_{17}\right.$ for $\left.\pi_{1 i}\right)$ & +0.0003 & 0.0002 & 0.16 & +0.0003 & 0.0003 & 0.34 & +0.0002 & 0.0003 & 0.49 \\
\hline Caffeine $\left(\gamma_{08}\right.$ for $\left.\pi_{0 i}\right)$ & -0.0001 & 0.001 & 0.88 & -0.0002 & 0.0008 & 0.83 & -0.0002 & 0.0008 & 0.84 \\
\hline Caffeine $\times$ Time $\left(\gamma_{18}\right.$ for $\left.\pi_{1 i}\right)$ & -0.0000 & 0.0001 & 0.92 & +0.0001 & 0.0002 & 0.70 & -0.0002 & 0.0002 & 0.40 \\
\hline
\end{tabular}

$n$, number of participants in the analysis; $n^{\prime}$, total number of visits included in the analysis; Age $_{\text {base, }}$, baseline age at visit 1

* Random effects are not shown for simplicity.

† Models were further adjusted for marital status, poverty status, education (years), baseline current smoking status, current illicit drug use and baseline BMl centred at $30 \mathrm{~kg} / \mathrm{m}^{2}$, the ten principal components for population structure, other dietary factors namely total grains, total fruit, total vegetables, other meats, discretionary solid fat and discretionary oils, and the inverse Mills ratio. Age base was centred at 50 years, sex was coded as $0=$ women, $1=$ men. All dietary factors were centred at their weighted means (see Table 1, total).

$\ddagger P<0.10$.

$\S P<0.05$

Passed correction for multiple testing.

I $P<0.05$ for interaction with sex to test effect modification by sex for each of the eight dietary factors on SUA at baseline and SUA change over time. 


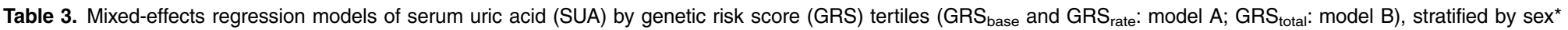
(Regression coefficients $(\gamma)$ with their standard errors of the estimate (SEE))

\begin{tabular}{|c|c|c|c|c|c|c|c|c|c|}
\hline \multirow[b]{3}{*}{ SUA } & \multicolumn{3}{|c|}{ Total† } & \multicolumn{3}{|c|}{ Men† } & \multicolumn{3}{|c|}{ Women† } \\
\hline & \multicolumn{2}{|c|}{$n 766$} & \multirow{2}{*}{$\frac{n^{\prime} 1341}{P}$} & \multicolumn{2}{|c|}{$n 343$} & \multirow{2}{*}{$\frac{n^{\prime} 583}{P}$} & \multicolumn{2}{|c|}{$n 423$} & \multirow{2}{*}{$\frac{n^{\prime} 758}{P}$} \\
\hline & $\gamma$ & SEE & & $\gamma$ & SEE & & $\gamma$ & SEE & \\
\hline \multirow{2}{*}{\multicolumn{10}{|c|}{$\begin{array}{l}\text { Model A: GRS base and rate } \\
\text { Fixed effects }\end{array}$}} \\
\hline & & & & & & & & & \\
\hline GRS $_{21 \text { base }}\left(\gamma_{021}\right.$ for $\left.\pi_{0 i}\right)$ & +0.32 & 0.11 & 0.004 & +0.36 & 0.18 & $0.043 \ddagger$ & +0.30 & 0.14 & 0.029 \\
\hline GRS $_{21 \text { rate }} \times$ Time $\left(\gamma_{121}\right.$ for $\left.\pi_{1 i}\right)$ & +0.02 & 0.020 & 0.47 & +0.03 & 0.041 & 0.42 & +0.02 & 0.03 & 0.56 \\
\hline $\mathrm{GRS}_{31 \text { base }}\left(\gamma_{031}\right.$ for $\left.\pi_{0 i}\right)$ & +0.54 & 0.12 & $<0.001$ & +0.44 & 0.18 & $0.013 \ddagger$ & +0.60 & 0.15 & $<0.001$ \\
\hline $\mathrm{GRS}_{31 \text { rate }} \times$ Time $\left(\nu_{131}\right.$ for $\left.\pi_{1 i}\right)$ & +0.06 & 0.03 & 0.037 & +0.11 & 0.05 & $0.021 \ddagger$ & +0.004 & 0.039 & 0.90 \\
\hline \multicolumn{10}{|l|}{ Model B: GRS total } \\
\hline \multicolumn{10}{|l|}{ Fixed effects } \\
\hline $\mathrm{GRS}_{21 \text { total }}\left(\gamma_{021}\right.$ for $\left.\pi_{0 i}\right)$ & +0.31 & 0.11 & 0.007 & +0.40 & 0.18 & 0.022 & +0.23 & 0.15 & 0.12 \\
\hline $\mathrm{GRS}_{21 \text { total }} \times$ Time $\left(\gamma_{121}\right.$ for $\left.\pi_{11}\right)$ & +0.01 & 0.03 & 0.73 & +0.02 & 0.05 & 0.61 & +0.02 & 0.03 & 0.56 \\
\hline $\mathrm{GRS}_{31 \text { total }}\left(\gamma_{031}\right.$ for $\left.\pi_{0 i}\right)$ & +0.50 & 0.12 & $<0.001$ & 0.44 & 0.19 & 0.020 & +0.56 & 0.16 & 0.001 \\
\hline $\mathrm{GRS}_{31 \text { total }} \times$ Time $\left(\gamma_{131}\right.$ for $\left.\pi_{11}\right)$ & +0.04 & 0.03 & 0.20 & 0.01 & 0.05 & 0.81 & +0.03 & 0.04 & 0.36 \\
\hline
\end{tabular}
$n$, number of participants in the analysis; $n^{\prime}$, total number of visits included in the analysis; Age $_{\text {base }}$, baseline age at visit 1; GRS $_{21}$, High Serum Uric Acid Risk Score dummy for tertile $2 v$. tertile 1; GRS ${ }_{31}$, High Serum Uric Acid Risk Score

dummy for tertile $3 \mathrm{v}$. tertile 1 .
* Random effects are not shown for simplicity.

† Models were further adjusted for marital status, poverty status, education (years), baseline current smoking status, current illicit drug use and baseline BMl centred at $30 \mathrm{~kg} / \mathrm{m}^{2}$, the ten principal components for population structure other dietary factors namely total grains, total fruit, total vegetables, other meats, discretionary solid fat and discretionary oils, and the inverse Mills ratio. Age dietary factors were centred at their weighted means (see Table 1, total). Tertiles of $G R S$. 15.18, mean values and standard deviations of GRS total $_{\text {within its tertiles. }}$

$\ddagger P<0.05$ for interaction with sex to test effect modification by sex for each of the two dummy variables (i.e. $\mathrm{GRS}_{21}$ and $\mathrm{GRS}_{31}$ ) on SUA at baseline and SUA change over time. 
Table 4. Mixed-effects regression models of serum uric acid (SUA) by dietary components, stratified by genetic risk score(GRS) tertile (GRS total $^{*}$ (Mean values and standard deviations; regression coefficients $(\gamma)$ with their standard errors of the estimate (SEE))

\begin{tabular}{|c|c|c|c|c|c|c|c|c|c|}
\hline $\mathrm{GRS}_{\text {total }}$ score & \multicolumn{3}{|c|}{$\mathrm{GRS}_{\text {total }}(\mathrm{T} 1)$} & \multicolumn{3}{|c|}{$\mathrm{GRS}_{\text {total }}(\mathrm{T} 2)$} & \multicolumn{3}{|c|}{$\mathrm{GRS}_{\text {total }}(\mathrm{T} 3)$} \\
\hline Mean & $9 \cdot 19$ & & & 13.51 & & & 16.88 & & \\
\hline SD & 2.05 & & & 1.09 & & & 1.37 & & \\
\hline \multirow[t]{3}{*}{ Range } & \multicolumn{3}{|c|}{$3-12$} & \multicolumn{3}{|c|}{$12-15$} & \multicolumn{3}{|c|}{$15-22$} \\
\hline & \multicolumn{3}{|c|}{ Model 1† } & \multicolumn{3}{|c|}{ Model 2† } & \multicolumn{3}{|c|}{ Model 3† } \\
\hline & \multicolumn{2}{|c|}{$n 256$} & $n^{\prime} 453$ & \multicolumn{2}{|c|}{ n 297} & $n^{\prime} 514$ & \multicolumn{2}{|c|}{$n 213$} & $n^{\prime} 374$ \\
\hline SUA & $r$ & SEE & $P$ & $\gamma$ & SEE & $P$ & $r$ & SEE & $P$ \\
\hline \multicolumn{10}{|l|}{ Fixed effects } \\
\hline Added sugar $\left(\nu_{01}\right.$ for $\left.\pi_{0 i}\right)$ & +0.005 & 0.063 & 0.94 & +0.004 & 0.006 & 0.55 & +0.004 & 0.008 & 0.65 \\
\hline Added sugar $\times$ Time $\left(\gamma_{11}\right.$ for $\left.\pi_{1 i}\right)$ & +0.002 & 0.001 & 0.15 & +0.001 & 0.002 & 0.59 & -0.002 & 0.002 & 0.21 \\
\hline Alcohol $\left(\gamma_{02}\right.$ for $\left.\pi_{0 i}\right)$ & +0.005 & 0.063 & 0.94 & $+0 \cdot 161 \ddagger$ & 0.061 & $0.009 \ddagger$ & $+0.091 \S$ & 0.047 & $0.05 \S$ \\
\hline Alcohol $\times$ Time $\left(\gamma_{12}\right.$ for $\left.\pi_{1 i}\right)$ & -0.005 & 0.013 & 0.71 & -0.012 & 0.02 & 0.48 & -0.015 & 0.012 & 0.19 \\
\hline Red meat $\left(\gamma_{03}\right.$ for $\left.\pi_{0 i}\right)$ & $+0.078 \ddagger$ & 0.031 & $0.011 \ddagger$ & +0.01 & 0.05 & 0.84 & -0.055 & 0.042 & $0.19 \|$ \\
\hline Red meat $\times$ Time $\left(\gamma_{13}\right.$ for $\left.\pi_{1 i}\right)$ & -0.005 & 0.006 & 0.37 & +0.02 & 0.01 & $0.22 \|$ & +0.005 & 0.010 & 0.58 \\
\hline Fish $\left(\gamma_{04}\right.$ for $\left.\pi_{0 i}\right)$ & +0.001 & 0.04 & 0.98 & $+0 \cdot 11 \ddagger$ & 0.05 & $0.021 \ddagger$ & -0.010 & 0.045 & 0.82 \\
\hline Fish $\times$ Time $\left(\gamma_{14}\right.$ for $\left.\pi_{1 i}\right)$ & -0.000 & 0.008 & 0.99 & -0.005 & 0.012 & 0.66 & -0.012 & 0.010 & 0.25 \\
\hline Legumes $\left(\gamma_{05}\right.$ for $\left.\pi_{0 i}\right)$ & -0.759 & 0.660 & 0.25 & -0.211 & 0.409 & 0.61 & $-0.49 \ddagger$ & 0.23 & $0.034 \ddagger$ \\
\hline Legumes $\times$ Time $\left(\gamma_{15}\right.$ for $\left.\pi_{1 i}\right)$ & $+0.491 \neq \Uparrow$ & 0.125 & $<0.001 \neq \pi$ & -0.038 & 0.102 & $0.71 \|$ & +0.083 & 0.050 & $0.10 \|$ \\
\hline Dairy products $\left(\gamma_{06}\right.$ for $\left.\pi_{0 i}\right)$ & -0.049 & 0.13 & 0.70 & +0.160 & 0.124 & 0.20 & $+0 \cdot 102$ & 0.139 & 0.46 \\
\hline Dairy products $\times$ Time $\left(\gamma_{16}\right.$ for $\left.\pi_{1 i}\right)$ & -0.013 & 0.024 & 0.61 & $-0.057 \S$ & 0.030 & $0.053 \S$ & -0.036 & 0.033 & 0.26 \\
\hline Vitamin $C\left(\gamma_{07}\right.$ for $\left.\pi_{0 i}\right)$ & +0.000 & 0.002 & 0.91 & -0.001 & 0.001 & 0.66 & $-0.005 \ddagger$ & 0.002 & $0.008 \ddagger$ \\
\hline Vitamin $C \times$ Time $\left(\gamma_{17}\right.$ for $\left.\pi_{1 i}\right)$ & +0.000 & 0.000 & 0.50 & +0.000 & 0.000 & 0.30 & +0.000 & 0.000 & 0.31 \\
\hline Caffeine $\left(\gamma_{08}\right.$ for $\left.\pi_{0 i}\right)$ & +0.001 & 0.001 & 0.17 & $-0.002 \S$ & 0.001 & $0.065 \S$ & -0.001 & 0.001 & 0.34 \\
\hline Caffeine $\times$ Time $\left(\gamma_{18}\right.$ for $\left.\pi_{1 i}\right)$ & -0.000 & 0.000 & 0.47 & +0.000 & 0.000 & 0.97 & +0.000 & 0.000 & 0.30 \\
\hline
\end{tabular}

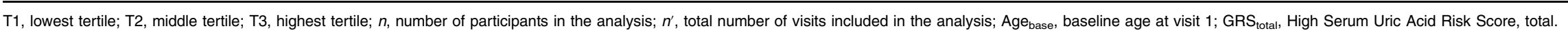
* Random effects are not shown for simplicity.

$\dagger$ Each of the model's intercepts and slopes were further adjusted for marital status, poverty status, education (years), baseline current smoking status, current illicit drug use and baseline BMI centred at $30 \mathrm{~kg} / \mathrm{m}^{2}$, the ten principal components for population structure, other dietary factors namely total grains, total fruit, total vegetables, other meats, discretionary solid fat and discretionary oils, and the inverse Mills ratio. Age $\mathrm{base}$ was centred at 50 years, sex was coded as $0=$ women, $1=$ men, and all dietary factors were centred at their weighted means (see Table 1 , total). $\neq P<0.05$.

॥ $P<0.10$.

I Passed correction for multiple testing. 
Table 5. Sex-specific interactions between genetic risk score (GRS) tertiles (GRS base $_{\text {and }}$ GRS risk $_{\text {) }}$ and dietary factors in their association with serum uric acid (SUA): mixed-effect regression models*

(Regression coefficients $(\gamma)$ with their standard errors of the estimate (SEE))

\begin{tabular}{|c|c|c|c|c|c|c|}
\hline \multirow[b]{3}{*}{ SUA } & \multicolumn{3}{|c|}{ Men† } & \multicolumn{3}{|c|}{ Woment } \\
\hline & \multicolumn{2}{|c|}{$n 343$} & \multirow{2}{*}{$\begin{array}{c}n^{\prime} 583 \\
P\end{array}$} & \multicolumn{2}{|c|}{$n^{\prime} 423$} & \multirow{2}{*}{$\begin{array}{c}n^{\prime} 758 \\
P\end{array}$} \\
\hline & $\gamma$ & SEE & & $\gamma$ & SEE & \\
\hline \multicolumn{7}{|l|}{ Added sugar } \\
\hline \multicolumn{7}{|l|}{ Model 1.A } \\
\hline Added sugar $\left(\gamma_{01}\right.$ for $\left.\pi_{0 i}\right)$ & +0.013 & 0.016 & 0.41 & +0.023 & 0.016 & 0.14 \\
\hline $\mathrm{GRS}_{\text {base }}\left(\gamma_{09}\right.$ for $\left.\pi_{0 i}\right)$ & $+0.093 \ddagger$ & 0.039 & $0.018 \ddagger$ & $+0 \cdot 126 \ddagger$ & 0.032 & $<0.001 \ddagger$ \\
\hline Added sugar $\times \mathrm{GRS}_{\text {base }}\left(\gamma_{019}\right.$ for $\left.\pi_{0 i}\right)$ & -0.001 & 0.01 & 0.57 & $-0.002 \S$ & 0.001 & $0.098 \S$ \\
\hline \multicolumn{7}{|l|}{ Model 1.B } \\
\hline Added sugar $\times$ Time $\left(\gamma_{11}\right.$ for $\left.\pi_{1 i}\right)$ & -0.000 & 0.001 & 0.91 & $-0.0026 \S$ & 0.0014 & $0.07 \S$ \\
\hline $\mathrm{GRS}_{\text {rate }} \times$ Time $\left(\nu_{19}\right.$ for $\left.\pi_{1 i}\right)$ & $+0.044 \ddagger$ & 0.019 & $0.018 \ddagger$ & -0.019 & 0.016 & 0.24 \\
\hline Added sugar $\times \mathrm{GRS}_{\text {rate }} \times$ Time $\left(\nu_{119}\right.$ for $\left.\pi_{1 i}\right)$ & +0.001 & 0.001 & 0.31 & +0.002 & 0.001 & 0.16 \\
\hline \multicolumn{7}{|l|}{ Alcohol } \\
\hline \multicolumn{7}{|l|}{ Model 2.A } \\
\hline Alcohol $\left(\gamma_{02}\right.$ for $\left.\pi_{0 i}\right)$ & -0.004 & 0.011 & 0.73 & -0.07 & 0.26 & 0.79 \\
\hline $\mathrm{GRS}_{\text {base }}\left(\gamma_{09}\right.$ for $\left.\pi_{0 i}\right)$ & $+0.073 \ddagger$ & 0.024 & $0.003 \ddagger$ & $+0.077 \ddagger$ & 0.019 & $<0.001 \ddagger$ \\
\hline Alcohol $\times \mathrm{GRS}_{\text {base }}\left(\gamma_{029}\right.$ for $\left.\pi_{0 i}\right)$ & +0.001 & 0.009 & 0.90 & +0.016 & 0.019 & 0.41 \\
\hline \multicolumn{7}{|l|}{ Model 2.B } \\
\hline Alcohol $\times$ Time $\left(\gamma_{12}\right.$ for $\left.\pi_{1 i}\right)$ & +0.004 & 0.011 & 0.73 & -0.018 & 0.014 & 0.13 \\
\hline $\mathrm{GRS}_{\text {rate }} \times$ Time $\left(\gamma_{19}\right.$ for $\left.\pi_{1 i}\right)$ & $+0.048 \ddagger$ & 0.019 & $0.010 \ddagger$ & +0.016 & 0.016 & 0.30 \\
\hline Alcohol $\times$ GRS $_{\text {rate }} \times$ Time $\left(\gamma_{129}\right.$ for $\left.\pi_{1 i}\right)$ & -0.012 & 0.011 & 0.31 & +0.001 & 0.011 & 0.90 \\
\hline Red meat & & & & & & \\
\hline Model 3.A & & & & & & \\
\hline Red meat $\left(\gamma_{03}\right.$ for $\left.\pi_{0 i}\right)$ & $+0 \cdot 13 \ddagger$ & 0.06 & $0.026 \ddagger$ & $+0.38 \ddagger$ & $0 \cdot 13$ & $0.003 \ddagger$ \\
\hline $\mathrm{GRS}_{\text {base }}\left(\gamma_{09}\right.$ for $\left.\pi_{0 i}\right)$ & $+0.095 \ddagger$ & 0.025 & $<0.001 \ddagger$ & $+0 \cdot 118 \ddagger$ & 0.023 & $<0.001 \ddagger$ \\
\hline Red meat $\times \mathrm{GRS}_{\text {base }}\left(\gamma_{039}\right.$ for $\left.\pi_{0 i}\right)$ & $-0 \cdot 010 \S$ & 0.06 & $0.09 \S$ & $-0.026 \ddagger$ & 0.010 & $0.014 \ddagger$ \\
\hline Model 3.B & & & & & & \\
\hline Red meat $\times$ Time $\left(\gamma_{13}\right.$ for $\left.\pi_{1 i}\right)$ & -0.001 & 0.008 & 0.86 & -0.003 & 0.010 & 0.80 \\
\hline $\mathrm{GRS}_{\text {rate }} \times$ Time $\left(\gamma_{19}\right.$ for $\left.\pi_{1 i}\right)$ & $+0.044 \ddagger$ & 0.018 & $0.014 \ddagger$ & +0.023 & 0.016 & 0.15 \\
\hline Red meat $\times$ GRS $_{\text {rate }} \times$ Time $\left(\gamma_{139}\right.$ for $\left.\pi_{1 i}\right)$ & +0.001 & 0.007 & 0.93 & $+0.025 \ddagger \|$ & 0.010 & $0.012 \neq \|$ \\
\hline Fish & & & & & & \\
\hline Model 4.A & & & & & & \\
\hline Fish $\left(\gamma_{04}\right.$ for $\left.\pi_{0 i}\right)$ & -0.018 & $0 \cdot 154$ & 0.91 & -0.002 & 0.087 & 0.98 \\
\hline $\mathrm{GRS}_{\text {base }}\left(\gamma_{09}\right.$ for $\left.\pi_{0 i}\right)$ & $+0.072 \ddagger$ & 0.024 & $0.003 \ddagger$ & $+0.077 \ddagger$ & 0.020 & $<0.001 \ddagger$ \\
\hline Fish $\times$ GRS $_{\text {base }}\left(\gamma_{049}\right.$ for $\left.\pi_{0 i}\right)$ & +0.003 & 0.014 & 0.82 & +0.004 & 0.007 & 0.57 \\
\hline Model 4.B & & & & & & \\
\hline Fish $\times$ Time $\left(\gamma_{14}\right.$ for $\left.\pi_{1 i}\right)$ & -0.003 & 0.010 & 0.76 & -0.006 & 0.007 & 0.39 \\
\hline $\mathrm{GRS}_{\text {rate }} \times$ Time $\left(\gamma_{19}\right.$ for $\left.\pi_{1 i}\right)$ & $+0.048 \ddagger$ & 0.019 & $0.010 \ddagger$ & +0.017 & 0.016 & 0.29 \\
\hline Fish $\times$ GRS $_{\text {rate }} \times$ Time $\left(\gamma_{149}\right.$ for $\left.\pi_{1 i}\right)$ & -0.006 & 0.008 & 0.46 & +0.001 & 0.008 & 0.90 \\
\hline Legumes & & & & & & \\
\hline Model 5.A & & & & & & \\
\hline Legumes $\left(\gamma_{05}\right.$ for $\left.\pi_{0 i}\right)$ & $+2 \cdot 146$ & $2 \cdot 315$ & 0.35 & $+1 \cdot 222$ & $1 \cdot 311$ & 0.35 \\
\hline $\mathrm{GRS}_{\text {base }}\left(\gamma_{09}\right.$ for $\left.\pi_{0 i}\right)$ & $+0.082 \ddagger$ & 0.023 & $<0.001 \ddagger$ & $+0.088 \ddagger$ & 0.019 & $<0.001 \ddagger$ \\
\hline Legumes $\times \mathrm{GRS}_{\text {base }}\left(\gamma_{059}\right.$ for $\left.\pi_{0 i}\right)$ & -0.219 & 0.202 & 0.28 & $-0 \cdot 102$ & 0.083 & 0.22 \\
\hline Model 5.B & & & & & & \\
\hline Legumes $\times$ Time $\left(\gamma_{15}\right.$ for $\left.\pi_{1 i}\right)$ & -0.056 & 0.091 & 0.54 & $+0.258 \ddagger$ & 0.077 & $0.001 \ddagger$ \\
\hline $\mathrm{GRS}_{\text {rate }} \times$ Time $\left(\gamma_{19}\right.$ for $\left.\pi_{1 i}\right)$ & $+0.048 \ddagger$ & 0.019 & $0.013 \ddagger$ & +0.007 & 0.016 & 0.66 \\
\hline Legumes $\times \mathrm{GRS}_{\text {rate }} \times$ Time $\left(\gamma_{159}\right.$ for $\left.\pi_{1 i}\right)$ & -0.073 & $0 \cdot 153$ & 0.63 & $+0 \cdot 219 \S$ & $0 \cdot 121 \S$ & $0.072 \S$ \\
\hline Dairy products & & & & & & \\
\hline Model 6.A & & & & & & \\
\hline Dairy products $\left(\gamma_{06}\right.$ for $\left.\pi_{0 i}\right)$ & -0.42 & 0.32 & 0.20 & $+0 \cdot 314$ & 0.290 & 0.28 \\
\hline $\mathrm{GRS}_{\text {base }}\left(\gamma_{09}\right.$ for $\left.\pi_{0 i}\right)$ & +0.035 & 0.033 & 0.29 & $+0.093 \ddagger$ & 0.025 & $<0.001 \ddagger$ \\
\hline Dairy products $\times \mathrm{GRS}_{\text {base }}\left(\gamma_{069}\right.$ for $\left.\pi_{0 i}\right)$ & $+0 \cdot 041$ & 0.026 & 0.12 & -0.015 & 0.026 & 0.55 \\
\hline Model 6.B & & & & & & \\
\hline Dairy products $\times$ Time $\left(\gamma_{16}\right.$ for $\left.\pi_{1 i}\right)$ & $-0.054 \ddagger$ & 0.027 & $0.043 \ddagger$ & $-0.053 \ddagger$ & 0.024 & $0.027 \ddagger$ \\
\hline GRS $_{\text {rate }} \times$ Time $\left(\gamma_{19}\right.$ for $\left.\pi_{1 i}\right)$ & $+0.043 \ddagger$ & 0.018 & $0.018 \ddagger$ & +0.019 & 0.016 & 0.23 \\
\hline Dairy products $\times \mathrm{GRS}_{\text {rate }} \times$ Time $\left(\gamma_{169}\right.$ for $\left.\pi_{1 i}\right)$ & +0.005 & 0.018 & 0.77 & +0.014 & 0.019 & 0.45 \\
\hline Vitamin C & & & & & & \\
\hline Model 7.A & & & & & & \\
\hline Vitamin $\mathrm{C}\left(\gamma_{07}\right.$ for $\left.\pi_{0 i}\right)$ & $-0.0121 \ddagger$ & 0.004 & $0.001 \ddagger$ & +0.002 & 0.003 & 0.51 \\
\hline $\mathrm{GRS}_{\text {base }}\left(\gamma_{09}\right.$ for $\left.\pi_{0 i}\right)$ & +0.0134 & 0.031 & $0 \cdot 66$ & $+0.097 \ddagger$ & 0.0263 & $<0.001 \ddagger$ \\
\hline Vitamin $\mathrm{C} \times \mathrm{GRS}_{\text {base }}\left(\gamma_{079}\right.$ for $\left.\pi_{0 i}\right)$ & $+0.001 \neq \|$ & 0.000 & $0.006 \ddagger 11$ & -0.0001 & 0.0002 & 0.47 \\
\hline Model 7.B & & & & & & \\
\hline Vitamin $\mathrm{C} \times$ Time $\left(\gamma_{17}\right.$ for $\left.\pi_{1 i}\right)$ & +0.0002 & 0.0003 & 0.79 & -0.0002 & 0.0003 & 0.58 \\
\hline $\mathrm{GRS}_{\text {rate }} \times$ Time $\left(\gamma_{19}\right.$ for $\left.\pi_{1 i}\right)$ & $+0.043 \ddagger$ & 0.018 & $0.018 \mp$ & +0.017 & 0.016 & 0.26 \\
\hline Vitamin $\mathrm{C} \times \mathrm{GRS}_{\text {rate }} \times$ Time $\left(\gamma_{179}\right.$ for $\left.\pi_{1 i}\right)$ & $-0.0005 \ddagger$ & 0.0002 & $0.026 \ddagger$ & +0.0003 & 0.0002 & $0 \cdot 19$ \\
\hline
\end{tabular}


Table 5. Continued

\begin{tabular}{|c|c|c|c|c|c|c|}
\hline \multirow[b]{3}{*}{ SUA } & \multicolumn{3}{|c|}{ Men† } & \multicolumn{3}{|c|}{ Women† } \\
\hline & \multicolumn{2}{|c|}{$n 343$} & \multirow{2}{*}{$\frac{n^{\prime} 583}{P}$} & \multicolumn{2}{|c|}{$n^{\prime} 423$} & \multirow{2}{*}{$\begin{array}{c}n^{\prime} 758 \\
P\end{array}$} \\
\hline & $\gamma$ & SEE & & $\gamma$ & SEE & \\
\hline \multicolumn{7}{|l|}{ Caffeine } \\
\hline \multicolumn{7}{|l|}{ Model 8.A } \\
\hline Caffeine $\left(\gamma_{08}\right.$ for $\left.\pi_{0 i}\right)$ & +0.002 & 0.002 & 0.51 & $+0.0048 \S$ & 0.028 & $0.083 \S$ \\
\hline $\mathrm{GRS}_{\text {base }}\left(\gamma_{09}\right.$ for $\left.\pi_{0 i}\right)$ & $+0.089 \ddagger$ & 0.029 & $0.002 \ddagger$ & $+0.111 \ddagger$ & 0.023 & $<0.001 \ddagger$ \\
\hline Caffeine $\times \mathrm{GRS}_{\text {base }}\left(\gamma_{089}\right.$ for $\left.\pi_{0 i}\right)$ & -0.0001 & 0.0002 & 0.46 & $-0.0004 \S$ & 0.0002 & $0.055 \S$ \\
\hline \multicolumn{7}{|l|}{ Model 8.B } \\
\hline Caffeine $\times$ Time $\left(\gamma_{18}\right.$ for $\left.\pi_{1 i}\right)$ & +0.0001 & 0.0002 & $0 \cdot 71$ & -0.0001 & 0.0002 & 0.48 \\
\hline GRS $_{\text {rate }} \times$ Time $\left(\gamma_{19}\right.$ for $\left.\pi_{1 i}\right)$ & $+0.046 \ddagger$ & 0.019 & $0.013 \ddagger$ & +0.020 & 0.016 & 0.21 \\
\hline Caffeine $\times$ GRS $_{\text {rate }} \times$ Time $\left(\gamma_{189}\right.$ for $\left.\pi_{1 i}\right)$ & -0.0001 & 0.0002 & 0.79 & $+0.0003 \S$ & 0.0002 & $0.055 \S$ \\
\hline
\end{tabular}

$n$, number of participants in the analysis; $n^{\prime}$, total number of visits included in the analysis; Age $_{\text {base, }}$, baseline age at visit 1.

* Random effects are not shown for simplicity.

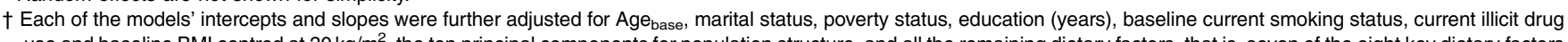
use and baseline BMI centred at $30 \mathrm{~kg} / \mathrm{m}^{2}$, the ten principal components for population structure, and all the remaining dietary factors, that is, seven of the eight key dietary factors in addition to total grains, total fruit, total vegetables, other meats, discretionary solid fat and discretionary oils, and the inverse Mills ratio. Age $e_{\text {base }}$ was centred at 50 years, and all

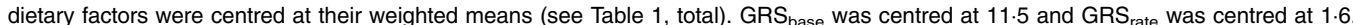

$\ddagger P<0.05$.

$\S P<0.10$

II Passed correction for multiple testing.

associations with SUA and gene-diet interactions. Among key findings, $\mathrm{SUA}_{\text {base }}$ was higher among men and increased with $\mathrm{GRS}_{\text {total }}$ tertiles. SUA rate was positively associated with legume intake among women $(\gamma+0 \cdot 14 ; 95 \% \mathrm{CI}+0 \cdot 06,+0 \cdot 22, P=0 \cdot 001)$ and inversely related to dairy product intake in both sexes combined $(\gamma=-0.042 ; 95 \%$ CI $-0.075,-0.009, \quad P=0.010)$. SUA $_{\text {base }}$ was directly linked to alcohol consumption among women $(\gamma+0 \cdot 154 ; 95 \% \mathrm{CI}+0 \cdot 046,+0 \cdot 262, P=0 \cdot 005) . \mathrm{GRS}_{\text {rate }}$ was linearly related to $\mathrm{SUA}_{\text {rate }}$ only among men. Legume consumption's positive association with $\mathrm{SUA}_{\text {rate }}$ was restricted to $\mathrm{GRS}_{\text {total }}$ 's lowest tertile. Among women, a synergistic interaction was observed between $\mathrm{GRS}_{\text {rate }}$ and red meat intake in association with $\mathrm{SUA}_{\text {rate }}$ Among men, a synergistic interaction between low vitamin $\mathrm{C}$ and genetic risk was also found.

Doring et al. indicated that the most significant SNP's associated with SUA were within the SLC2A9 gene, introns 4 and 6 $\left(P<1.2 \times 10^{-8}\right)$. This gene encodes two GLUT9 isoforms of the class II facilitative glucose transport family ${ }^{(16)}$. The long isoform GLUT9a (SLC2A9_L, GLUT9, 540 amino acids) is strongly expressed among humans in the basolateral side of the proximal renal tubular cells, and is responsible for the transport of UA back into the bloodstream ${ }^{(33)}$, whereas the shorter isoform GLUT9b (SLC2A9_S, GLUT9 $\Delta \mathrm{N}, 511$ amino acids) is expressed only in the apical membrane of polarised renal tubular cells, and gain of function mutations would be expected to increase the reuptake of excreted UA causing hyperuricaemia $^{(34)}$. Although RNA expression analysis has confirmed that the short isoform of $S L C 2 A 9$ was significantly and positively associated with SUA, to our knowledge SNP associated with $S L C 2 A 9 b$ have yet to predict amino acid changes in GLUT9b which would predict a gain of function. Conversely, loss of function mutations in $S L C 2 A 9 b$ have been reported to be causative of renal hypouricaemia in human subjects ${ }^{(16,35,36)}$. However, none of the SLC2A9 SNP are predicted to be deleterious in in silico functional annotation. Experimental studies are required to assess the biological consequences of these variants.
Fructose is also a substrate for liver GLUT9a (the longer isoform), as well as GLUT5 and GLUT11 ${ }^{(16)}$. Following its transport into hepatocytes, fructose is phosphorylated by fructokinase, generating ADP that is rapidly transformed into $\mathrm{UA}^{(16)}$. Therefore, the net effect of increasing fructose intake would be facilitative of liver purine breakdown into UA, thus increasing SUA ${ }^{(24)}$.

In a large GWAS by Kolz et al., the rs734553 minor allele in SLC $2 A 9$ had a stronger effect on reducing SUA in women, while the effect was stronger in men for the minor allele of rs2231142 in ABCG2 which elevates SUA ${ }^{(17)}$. The percentage variance explained by $S L C 2 A 9$ variants in SUA differs between sexes with genotypes explaining $1.2 \%$ in men and $6 \%$ in women and expression levels explaining $3.5 \%$ in men and $15 \%$ in women ${ }^{(16)}$. Another confirmatory study genotyped four previously identified SNP in the SLC2A9 gene (rs6855911, rs7442295, rs6449213 and rs12510549) and found significant associations with SUA in the expected direction. However, this association was significantly stronger among women and among individuals with higher $\mathrm{BMI}^{(18)}$. Our study indicated that the uppermost tertile of $\mathrm{GRS}_{\text {base }}$ was more strongly associated with $\mathrm{SUA}_{\text {base }}$ in women compared with men, though the reverse was true for the middle tertile. However, GRS rate was positively linked to $\mathrm{SUA}_{\text {rate }}$ only in men, while comparing the uppermost tertile to the lowest. As most other studies were cross-sectional and considering the uppermost tertile $v$. lowest contrast as the most important finding, our results replicated those prior studies, particularly that $\mathrm{GRS}_{\text {base }}$ consisted mostly of SLC2A9 gene SNP ${ }^{(16-18)}$.

The association between diet and SUA were also explored in previous studies, though failing to test sex-related differences. Given the consistently higher levels of SUA in men compared with that in women, it is important to include sex as an effect modifier when examining other risk factors for SUA levels. Large prospective cohort studies showed that higher meat and seafood intakes were associated with higher gout risk and higher SUA concentrations ${ }^{(1,8)}$. However, no association was detected for other purine-rich foods including peas, lentils, 
(a)

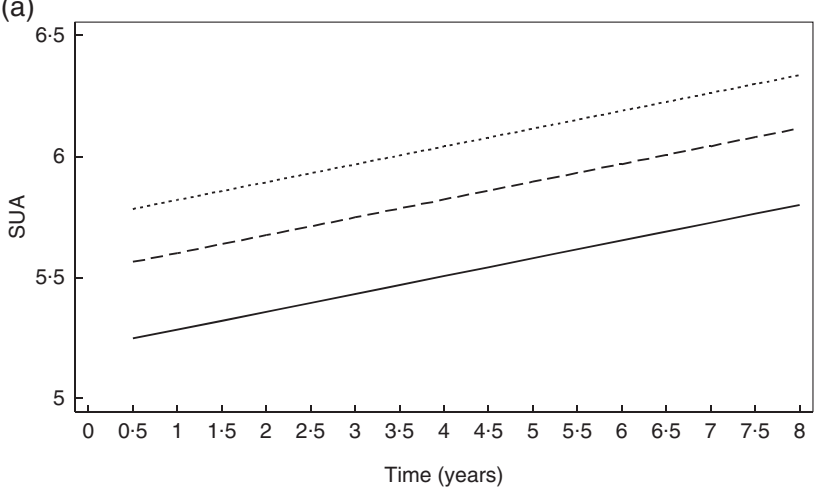

(b)

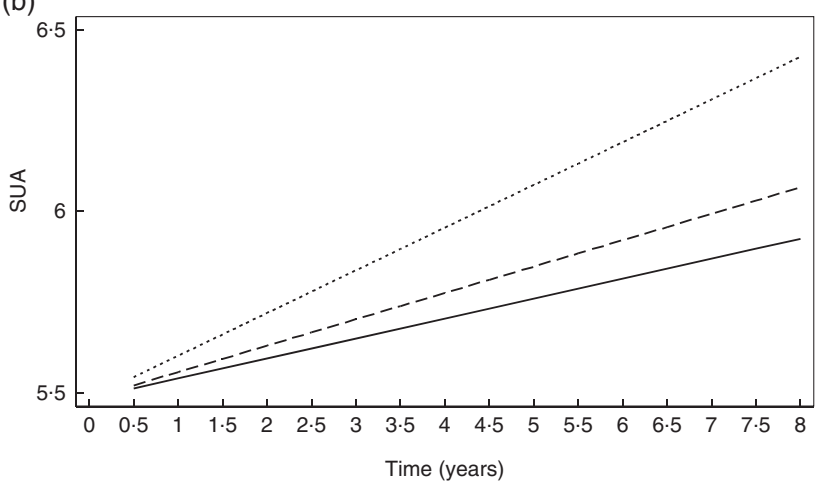

Fig. 1. Predictive margins of serum uric acid (SUA) by time and tertiles (T) of genetic risk scores (GRS), (a) GRS base and (b) GRS rate, $_{\text {, from mixed-effects }}$ regression model, total population. Predictive margins obtained from mixed-effects regression model with SUA as the outcome, random effects added to slope and intercept, and both slopes and intercept adjusted for multiple factors including age, sex, poverty status, marital status, education, smoking and drug use, several dietary factors, BMI, ten principal components for population structure and an inverse Mills ratio. The figure simulates the trajectory of a population with comparable characteristics (covariates set at their observed values in the sample) when exposed alternatively to $\mathrm{T} 1, \mathrm{~T} 2$ and $\mathrm{T} 3$ of $\mathrm{GRS}_{\text {base }}$ and $\mathrm{GRS}_{\text {rate, }}$ respectively (see Table 3, model 1). (a): —_, GRS base, $_{1}$ T1 . . . . ., GRS base, T2; ............,

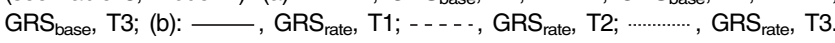
Tertiles of GRS base had the following distribution: T1 ( $n$ 258, mean 7.80, SD 1.95, range 2-10); T2 ( $n 279$, mean 11.76, SD 0.94, range 10-13); T3 ( $n$ 229, mean 15.18, SD 1.28, range 13-19). Tertiles of GRS rate had the following distribution: T1 ( $n 325$, mean 0.68 , SD 0.46 , range $0-1$ ); T2 ( $n$ 291, mean 1.85 , SD 0.34 , range $1-2)$; Т3 ( $n$ 150, mean 2.88, SD 0.61 , range $2-5$ ).

beans, spinach, mushrooms and cauliflower ${ }^{(1)}$, highlighting the importance of amount, bioavailability and types of purines in foods $^{(1)}$. We found that among women, there was a synergistic interaction between $\mathrm{GRS}_{\text {rate }}$ and red meat intake in association

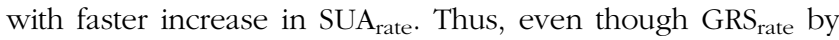
itself was not associated with $\mathrm{SUA}_{\text {rate }}$ among AA women (unlike among AA men), red meat consumption in this group may accelerate the genetic risk's effect on SUA's rate of increase. In other words, there is a super-additive effect of increasing meat consumption and increasing genetic risk on the rate of change in SUA among women. The biological mechanism behind this finding is worth further exploration. Furthermore, randomised controlled trials of red meat consumption in relation $\mathrm{SUA}_{\text {rate }}$ should be conducted among AA women while stratifying by genetic risk, to replicate those findings.

The positive association between legume consumption with $\mathrm{SUA}_{\text {rate }}$ was restricted to the lowest tertile of $\mathrm{GRS}_{\text {total }}$, indicating an antagonistic interaction, and was significantly stronger in women. Thus, legume consumption may affect the rate at which SUA increases over time in women and among individuals with lower genetic risk for elevated SUA. This finding is novel and worth further exploration in larger AA adult samples, particularly that the positive association between legume intake and SUA was only found in animal studies ${ }^{(15)}$.

Fructose intake, as discussed earlier, exerts a direct effect on SUA, through liver ATP utilisation for phosphorylation and production of ADP. In addition, SLC2A9 transports both fructose and UA with maximal transport of fructose occurring in the absence of UA. In fact, oral fructose administration in hyperuricaemic patients further increased SUA ${ }^{(1,37)}$. Using national data (National Health and Nutrition Examination Survey III (NHANES III)) on 14761 adults, soft drink consumption was shown to increase SUA in a dose-response way from $+0.08 \mathrm{mg} / \mathrm{dl}$ higher SUA (for $<0.5$ servings $v$. no intake), to $0.42 \mathrm{mg} / \mathrm{dl}$ higher SUA (for $\geq 4$ servings $/ \mathrm{d} v$. no intake), $P_{\text {for trend }}=0 \cdot 003$. Findings were similar for sugar-sweetened soft drinks in relation to the odds of hyperuricaemia ${ }^{(11)}$, and were replicated only in men in another analysis using NHANES 2001-2002 ${ }^{(12)}$. At least one study found a non-additive interaction between SLC2A9 genotype and sugar-sweetened beverage consumption in determining the risk for gout, when analysing genotype-specific groups $^{(38)}$. Our study did not detect an association between added sugars and $\mathrm{SUA}_{\text {base/rate, possibly due to differences }}$ between our study and previous ones in terms of racial/ethnic composition. However, larger studies of AA adult populations are needed to replicate those findings.

Based on a recent meta-analysis of 42924 adults, alcohol consumption had a linear dose-response relationship with gout. Compared with no/little alcohol drinking, light ( $\leq 1 \mathrm{drink} / \mathrm{d})$, moderate $(>1-<3$ drinks/d) and heavy drinking $(\geq 3$ drinks $/ \mathrm{d})$ had a risk ratio of 1.16 (95\% CI 1.07, 1.25), 1.58 (95\% CI 1.50 , 1.66 ) and 2.64 (95\% CI 2.26, 3.09), respectively ${ }^{(39)}$. Studies also indicated that the association between alcohol and SUA pertained mostly to beer and liquor/spirits ${ }^{(7)}$. Similar to fructose, alcohol increases liver UA production through ATP degradation, leading to accumulation of ADP and AMP. Alcohol intake additionally leads to dehydration and metabolic acidosis, resulting in a decreased urate excretion ${ }^{(1)}$. A study among Japanese adults confirmed an association between SUA and an LDL-receptor-related protein (LRP2) polymorphism rs2544390 $(\mathrm{C} / \mathrm{T})$. The study found this association to be stronger among males drinking five times or more per week, with a significant gene-diet interaction, indicating synergism ${ }^{(40)}$. In contrast, an antagonistic interaction on gout outcomes was found in another study that combined Maori and Pacific Islanders, in which alcohol consumption was associated with higher risk for gout only in the rs2544390 CC genotype group ${ }^{(41)}$. Another study showed alcohol consumption and $A B C G 2$ Q141K was independently and jointly associated with the risk for chronic tophaceous gout ${ }^{(42)}$. Our findings indicated sex-specific associations between alcohol and SUA (stronger cross-sectional positive effect in women), without detecting any gene-diet interactions. This suggests that among women, reducing alcohol consumption may potentially reduce SUA, irrespective of genetic risk for elevated SUA. 
Vitamin C may also reduce SUA based on a cross-sectional study ${ }^{(43)}$ and a meta-analysis of randomised controlled trials that administered a median dose of $500 \mathrm{mg} / \mathrm{d}^{(44)}$. Biological mechanisms involved include a uricosuric effect of vitamin $\mathrm{C}$ at the URAT1 and a sodium-dependent anion co-transporter solute carrier family 5, member 8 (SLCA5A8)/A12; an enhanced fractional kidney clearance of UA; and a reduced oxidative damage of body cells which reduces SUA ${ }^{(14)}$. In our study, among men, low vitamin $\mathrm{C}$ was shown to increase $\mathrm{SUA}_{\text {base }}$ only at higher $\mathrm{GRS}_{\text {base }}$ levels, indicating a synergistic interaction. This suggests that among AA men, increasing intake of vitamin $\mathrm{C}$ may potentially reduce $\mathrm{SUA}_{\text {base, }}$, particularly when genetic risk is elevated. However, randomised controlled trials among AA men and stratified by genetic risk are needed to confirm this observation.

Several studies have shown a relationship between dairy product consumption and SUA/gout ${ }^{(10,14)}$. The evidence thus far points to a protective effect of milk and low-fat yogurt against gout occurrence and hyperuricaemia ${ }^{(8)}$. There is also evidence that a vegan diet lacking dairy products is more hyperuricaemic than a vegetarian or fish eating type of diet, with the differences most pronounced among men ${ }^{(45)}$. Several mechanisms were suggested including the effects of orotic acid in milk which promotes renal urate excretion, the uricosuric effect of milk casein and lactalbumin, and a putative biological effect of vitamin D on SUA which has yet to be confirmed $^{(14)}$. Besides specific dietary components, a higher Mediterranean diet score was linked to lower SUA ${ }^{5,6}$, particularly among women ${ }^{(5)}$. We found that $\mathrm{SUA}_{\text {rate }}$ was inversely related to dairy product intake in the overall AA sample (Dairy product $\times$ Time effect: $\gamma-0.042$ (SEE 0.017), $P=0.010$ ). However, there were no gene-diet or sex-diet interactions for this dietary component.

Among its strengths, this study systematically examined SNP previously shown to be associated with higher SUA and evaluated SUA's sex-specific association with a composite GRS, while testing gene-diet interactions. Our study is among the few to include AA. Despite its strengths, some limitations include a statistical power-limiting small sample size, which precluded further adjustment for incomplete potential confounders such as lipid profiles, ferritin, C-reactive protein and depressive symptoms. In fact, further analyses suggested that the power to detect the effect that was detected in our models was more adequate for the total population than for sexstratified models. Another limitation is the lack of adequately measured baseline covariates that could potentially act as confounders, including baseline physical activity. Moreover, most of our selected SNP came from studies conducted among subjects of European ancestry as well as other ethnic groups because of the paucity of studies among AA. Availability of genetic data in our HANDLS study among Whites would have strengthened our findings if replicated. Moreover, although GRS weighting by effect size was possible, we opted not to weight our gene scores due to the multiplicity of racial and ethnic groups in previous studies and for ease of interpretation. Finally, because of the low level of correlation between dietary factors that were related to SUA $(r<0 \cdot 20)$, a valid index for elevated SUA or faster increase in SUA could not be computed.
In sum, sex-diet, sex-gene and gene-diet interactions were detected in determining SUA. Dietary factors which interacted with genetic risk to alter $\mathrm{SUA}_{\text {base/rate }}$ included legumes (overall), red meat (among women) and vitamin C (among men). Legumes and alcohol intakes were shown to potentially alter SUA's trajectory only in women. Finally, the GRS rate altered the rate of change in SUA only among men. Further studies on similar AA adult populations and incorporating larger samples of men and women are needed to replicate our findings.

\section{Acknowledgements}

The authors would like to thank Gregory A. Dore and Ola S. Rostant for their internal review of the manuscript.

This work was fully supported by the Intramural Research Program of the NIH, National Institute on Aging.

M. A. B.: had full access to the data used in this paper, performed data management and all statistical analyses and had primary responsibility for the final content; J.-A. C. and M. T. F.-K.: participated in the literature review; M. A. B., J.-A. C., M. T. F.-K., S. M. T., M. K. E. and A. B. Z.: wrote and revised the manuscript; M. A. B., J-A. C., M. T. F.-K. and A. B. Z.: participated in the plan of analysis; M. T. F.-K., M. K. E. and A. B. Z.: participated in data acquisition; S. M. T.: participated in data management and statistical analysis. All authors read and approved the final version of the manuscript.

None of the authors has any conflicts of interest to declare.

\section{Supplementary material}

For supplementary material/s referred to in this article, please visit https://doi.org/10.1017/S0007114517000411

\section{References}

1. Choi H, Mount D \& Reginato A (2005) Pathogenesis of gout. Ann Intern Med 143, 499-516.

2. George RL \& Keenan RT (2013) Genetics of hyperuricemia and gout: implications for the present and future. Curr Rheumatol Rep 15, 309.

3. Fang J \& Alderman M (2000) Serum uric acid and cardiovascular mortality the NHANES I epidemiologic follow-up study, 1971-1992. National Health and Nutrition Examination Survey. JAMA 283, 2404-2410.

4. Merriman TR (2015) An update on the genetic architecture of hyperuricemia and gout. Arthritis Res Ther 17, 98.

5. Kontogianni MD, Chrysohoou C, Panagiotakos DB, et al. (2012) Adherence to the Mediterranean diet and serum uric acid: the ATTICA study. Scand J Rheumatol 41, 442-449.

6. Chrysohoou C, Skoumas J, Pitsavos C, et al. (2011) Longterm adherence to the Mediterranean diet reduces the prevalence of hyperuricaemia in elderly individuals, without known cardiovascular disease: the Ikaria study. Maturitas $\mathbf{7 0}$, 58-64.

7. Choi HK, Atkinson K, Karlson EW, et al. (2004) Alcohol intake and risk of incident gout in men: a prospective study. Lancet 363, 1277-1281.

8. Choi HK, Atkinson K, Karlson EW, et al. (2004) Purine-rich foods, dairy and protein intake, and the risk of gout in men. N Engl J Med 350, 1093-1103. 
9. Torralba KD, De Jesus E \& Rachabattula S (2012) The interplay between diet, urate transporters and the risk for gout and hyperuricemia: current and future directions. Int J Rheum Dis 15, 499-506.

10. Poletto J, Harima HA, Ferreira SR, et al. (2011) Hyperuricemia and associated factors: a cross-sectional study of JapaneseBrazilians. Cad Saude Publica 27, 369-378.

11. Choi JW, Ford ES, Gao X, et al. (2008) Sugar-sweetened soft drinks, diet soft drinks, and serum uric acid level: the Third National Health and Nutrition Examination Survey. Arthritis Rheum 59, 109-116.

12. Gao X, Qi L, Qiao N, et al. (2007) Intake of added sugar and sugar-sweetened drink and serum uric acid concentration in US men and women. Hypertension 50, 306-312.

13. Gaffo AL, Roseman JM, Jacobs DR, et al. (2010) Serum urate and its relationship with alcoholic beverage intake in men and women: findings from the Coronary Artery Risk Development in Young Adults (CARDIA) cohort. Ann Rheum Dis 69, 1965-1970.

14. Towiwat P \& Li ZG (2015) The association of vitamin C, alcohol, coffee, tea, milk and yogurt with uric acid and gout. Int J Rheum Dis 18, 495-501.

15. Rotimi SO, Olayiwola I, Ademuyiwa O, et al. (2010) Inability of legumes to reverse diabetic-induced nephropathy in rats despite improvement in blood glucose and antioxidant status. $J$ Med Food 13, 163-169.

16. Doring A (2008) SLC2A9 influences uric acid concentrations with pronounced sex-specific effects. Nat Genet $\mathbf{4 0}$, 430-436.

17. Kolz M (2009) Meta-analysis of 28,141 individuals identifies common variants within five new loci that influence uric acid concentrations. PLoS Genet 5, e1000504.

18. Brandstatter A (2008) Sex-specific association of the putative fructose transporter SLC2A9 variants with uric acid levels is modified by BMI. Diabetes Care 31, 1662-1667.

19. Evans MK, Lepkowski JM, Powe NR, et al. (2010) Healthy Aging in Neighborhoods of Diversity Across the Life Span (HANDLS): overcoming barriers to implementing a longitudinal, epidemiologic, urban study of health, race, and socioeconomic status. Ethn Dis 20, 267-275.

20. Moshfegh AJ, Rhodes DG, Baer DJ, et al. (2008) The US Department of Agriculture Automated Multiple-Pass Method reduces bias in the collection of energy intakes. Am J Clin Nutr 88, 324-332.

21. US Department of Agriculture Agricultural Research Service \& Food Surveys Research Group (2016) USDA Food and Nutrient Database for Dietary Studies, 3.0. http://www.ars. usda.gov/Services/docs.htm?docid=12089 (accessed February 2017).

22. Reginato AM, Mount DB, Yang I, et al. (2012) The genetics of hyperuricaemia and gout. Nat Rev Rheumatol 8, 610-621.

23. Voruganti VS, Laston S, Haack K, et al. (2015) Serum uric acid concentrations and SLC2A9 genetic variation in Hispanic children: the Viva La Familia Study. Am J Clin Nutr 101, 725-732.

24. Yang B, Mo Z, Wu C, et al. (2014) A genome-wide association study identifies common variants influencing serum uric acid concentrations in a Chinese population. BMC Med Genomics 7, 10 .

25. Li C, Yu Q, Han L, et al. (2014) The hURAT1 rs559946 polymorphism and the incidence of gout in Han Chinese men. Scand I Rheumatol 43, 35-42.
26. Kottgen A (2013) Genome-wide association analyses identify 18 new loci associated with serum urate concentrations. Nat Genet 45, 145-154.

27. Lohr SL (1999) Sampling: Design and Analysis. Boston, MA: Duxbury-Press.

28. Ibrahim JG \& Molenberghs G (2009) Missing data methods in longitudinal studies: a review. Test (Madr) 18, 1-43.

29. Selvin S (2004) Statistical Analysis of Epidemiologic Data, 3rd ed. New York: Oxford University Press.

30. Hochberg Y \& Tamhane AC (1987) Multiple Comparison Procedures. New York: Wiley.

31. Beydoun MA, Beydoun HA, Kitner-Triolo MH, et al. (2013) Thyroid hormones are associated with cognitive function: moderation by sex, race, and depressive symptoms. J Clin Endocrinol Metab 98, 3470-3481.

32. Beydoun MA, Beydoun HA, Rostant OS, et al. (2015) Thyroid hormones are associated with longitudinal cognitive change in an urban adult population. Neurobiol Aging 36, 3056-3066.

33. Witkowska K, Smith KM, Yao SY, et al. (2012) Human SLC2A9a and SLC2A9b isoforms mediate electrogenic transport of urate with different characteristics in the presence of hexoses. Am J Physiol Renal Physiol 303, F527-F539.

34. Augustin R, Carayannopoulos MO, Dowd LO, et al. (2004) Identification and characterization of human glucose transporter-like protein-9 (GLUT9): alternative splicing alters trafficking. J Biol Chem 279, 16229-16236.

35. Anzai N, Kanai Y \& Endou H (2007) New insights into renal transport of urate. Curr Opin Rheumatol 19, 151-157.

36. Matsuo H, Chiba T, Nagamori S, et al. (2008) Mutations in glucose transporter 9 gene SLC2A9 cause renal hypouricemia. Am J Hum Genet 83, 744-751.

37. Emmerson BT (1974) Effect of oral fructose on urate production. Ann Rheum Dis 33, 276-280.

38. Batt C, Phipps-Green AJ, Black MA, et al. (2014) Sugarsweetened beverage consumption: a risk factor for prevalent gout with SLC2A9 genotype-specific effects on serum urate and risk of gout. Ann Rbeum Dis 73, 2101-2106.

39. Wang M, Jiang X, Wu W, et al. (2013) A meta-analysis of alcohol consumption and the risk of gout. Clin Rheumatol 32, 1641-1648.

40. Hamajima N, Naito M, Okada R, et al. (2012) Significant interaction between LRP2 rs2544390 in intron 1 and alcohol drinking for serum uric acid levels among a Japanese population. Gene 503, 131-136.

41. Rasheed H, Phipps-Green A, Topless R, et al. (2013) Association of the lipoprotein receptor-related protein 2 gene with gout and non-additive interaction with alcohol consumption. Arthritis Res Ther 15, R177.

42. Tu HP, Ko AM, Chiang SL, et al. (2014) Joint effects of alcohol consumption and ABCG2 Q141K on chronic tophaceous gout risk. J Rheumatol 41, 749-758.

43. Ryu KA, Kang HH, Kim SY, et al. (2014) Comparison of nutrient intake and diet quality between hyperuricemia subjects and controls in Korea. Clin Nutr Res 3, 56-63.

44. Juraschek SP, Miller ER 3rd \& Gelber AC (2011) Effect of oral vitamin $\mathrm{C}$ supplementation on serum uric acid: a metaanalysis of randomized controlled trials. Arthritis Care Res (Hoboken) 63, 1295-1306

45. Schmidt JA, Crowe FL, Appleby PN, et al. (2013) Serum uric acid concentrations in meat eaters, fish eaters, vegetarians and vegans: a cross-sectional analysis in the EPIC-Oxford cohort. PLOS ONE 8, e56339. 